\title{
Chirurgie des Nasenseptums
}

Marc Scheithauer, Janina Hahn, Marlene C. Wigand

Die Septumplastik zur Verbesserung der Nasenatmung ist eine der häufigsten Operationen in der HNO. Das Verständnis der chirurgischen Anatomie der Nase und die Kenntnis der standardisierten Operationssequenzen einer SPL stellen die theoretische Basis für diesen Eingriff dar. Erst wenn der junge Chirurg eine SPL beherrscht, ist es ihm möglich, Deformitäten der Außennase, im Sinne einer Septorhinoplastik, zu behandeln.

\section{Einleitung}

Die Septumplastik (SPL) als eine der häufigsten Operationen in der HNO ist in der Weiterbildungsordnung zur Erlangung des Facharztstatus als „Ausbildungseingriff“ hinterlegt. Das Verständnis der chirurgischen Anatomie der Nase und die Kenntnis der standardisierten Operationssequenzen einer SPL stellen die theoretische Basis für diesen Eingriff dar. Mehr noch: Erst wenn der junge Chirurg eine SPL beherrscht, ist es ihm möglich, das nächste Niveau der Rhinochirurgie, also Deformitäten der Außennase im Sinne einer Septorhinoplastik, zu behandeln. Dazu benötigt man viel Erfahrung, ein gutes ästhetisches Empfinden und Ausdauer. Die Lernphase erstreckt sich über das gesamte Berufsleben, und dennoch kann Perfektion nie erreicht werden.

\section{Die moderne Septumplastik}

Die moderne Septumplastik existiert seit 1948 und ist untrennbar mit dem Namen Maurice Cottle verbunden, einem damaligen Rhinochirurgen aus Chicago. Er hatte die Idee eines neuen operativen Zugangs, der es ermöglichte, alle Septumareale übersichtlich zu exponieren. Durch die Hemitransfixionsinzision können alle relevanten Landmarken (kaudale Septumkante, Spina nasalis anterior, anterior-inferiore Septumkante) zuverlässig dargestellt werden. Über den „maxillär-prämaxillären“ Zugang ist der Einstieg in die beiden vorderen oberen und unteren Tunnel simultan möglich. Cottle erkannte auch, dass der knorpeligen Lamina quadrangularis des Septums die wesentliche Stützfunktion zugeordnet werden muss. Dabei bestimmen die Dimension, die Position und die Stabilität der kaudalen Septumkante im Wesentlichen Funktion und Ästhetik der Nase ( $\triangleright$ Abb. 1).

Damit ist das zentrale Dogma der Septumplastik (SPL) formuliert. Eine SPL ist immer dann indiziert, wenn durch die Untersuchung der inneren und äußeren Nase eine anatomische Pathologie gefunden wird, die die subjektiv beklagte Nasenatmungsbehinderung mit hoher Wahrscheinlichkeit verursacht und erklärt. Im Rahmen der präoperativen Analyse ist die Endoskopie der Innennase ein unverzichtbarer Bestandteil der Diagnostik, da z. B. eine hohe Septumdeviation mit Einengung des Nasenklappenwinkels nur bei dieser Untersuchung sicher erkannt wird.

Merke

Ziel ist die Herstellung einer „balancierten Spannung“ im knorpeligen Septum mit ausreichend weiten Spalträumen vor allem im Naseneingang und auf Höhe des Nasenklappenwinkels. Aufgrund der verschiedenen benachbarten anatomischen Strukturen, die untereinander unmittelbar in Kontakt stehen, bestimmt das knorpelige Septum die Position, die Form und die Stabilität der Nasenspitze. Außerdem werden dadurch die Form der Nasenlöcher, die Stellung und die Breite der Kolumella, der Winkel der Nasenklappe und schließlich auch Form und Höhe des knorpeligen Nasenrückens definiert.

Dieser besteht aus der Einheit von dorsalem Septumknorpel und den lateral daran fusionierten beiden Seitenknorpeln („septodorsaler Komplex“).

\section{Operatives Vorgehen}

Die SPL umfasst standardisiert nachfolgende 6 operative Sequenzen:

1. Inzision $=$ Hemitransfixion

2. Zugang = subperichondrale und/oder subperiostale Tunnel

3. Mobilisation durch Chondrotomien und/oder Osteotomien

4. Resektion von Knorpel und/oder Knochen

5. Replantation von extrakorporal begradigtem Material/

Rekonstruktion des kaudalen Septums

6. Naht der Inzision und Fixation (Septumfolien) 


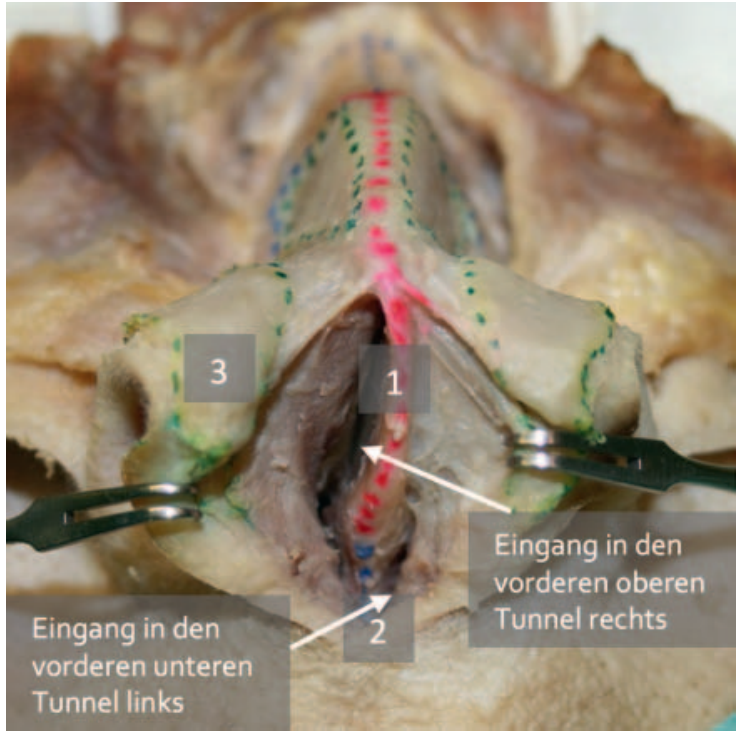

- Abb. 1 Anatomisches Präparat mit Darstellung der subperichondral angelegten Tunnel. 1 kaudale Septumkante; $\mathbf{2}$ Prämaxilla mit der Spina nasalis anterior; 3 Flügelknorpel.

Als vorbereitende Maßnahmen vor der Inzision sollten die Schleimhautabschwellung (Xylometazolin-haltige Schwämmchen), die Infiltrationsanästhesie (Scandicain/ Adrenalin 1:200 000) sowie die Reinigung der Naseneingänge (Octenisept) erfolgen.

\section{Inzision}

Die Standard-Inzision ist die Hemitransfixionsinzision, die bei Rechtshändern in der Vestibulumhaut im rechten Naseneingang mit einem Skalpell Nr. 15 geführt wird. Das Spekulum spannt die Haut über der kaudalen Septumkante, die anschließende Schnittführung erfolgt von kaudal (= Höhe der Spina nasalis anterior) nach kranial (= Höhe des anterior-inferioren Septumwinkels). Der Knorpel darf dabei nicht inzidiert werden, da sonst die stabilisierende Integrität der kaudalen Septumkante gefährdet wird. Drei Landmarken sollten nach der Inzision erkennbar sein: 1. kaudale Septumkante, 2. Spina nasalis anterior, 3. anterior-inferiorer Septumwinkel ( $\triangleright$ Abb. 2).

\section{Zugang}

Hierbei gilt: Je komplexer die Septumdeviation, desto mehr Tunnel sollten aus Gründen der Übersichtlichkeit angelegt werden.

\section{MERKE}

Die korrekte Präparationsebene ist die gefäßfreie subperichondrale Schicht, in die möglichst unmittelbar nach Darstellung der kaudalen Septumkante eingegangen werden sollte.

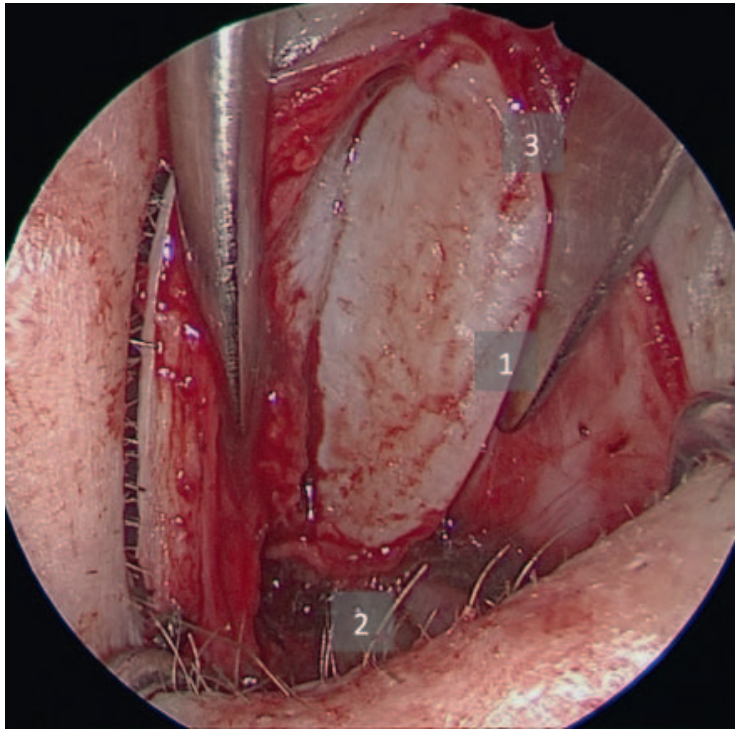

- Abb. 2 Hemitransfixionsinzision und Darstellung der (1) kaudalen Septumkante, der (2) Spina nasalis inferior und des (3) anterior-inferioren Septumwinkels.

Die Spitze der geschlossenen Schere ist dabei hilfreich und schont bei tangentialer Präparation den Knorpel. Etwa $1 \mathrm{~cm}$ posterior der kaudalen Septumkante sollte die Anlage der Tunnel mit dem Präpariersauger fortgeführt werden. Das in den Tunnel eingeführte Spekulum trennt in der Tiefe die Schleimhaut vom Knorpel. Über dem Septumknochen (=Lamina perpendicularis) empfiehlt sich für die weitere Präparation die Verwendung des schlanken Cottle-Elevatoriums. Bei einer einseitigen, anterior gelegenen Deviation wird empfohlen, mit dem Tunnel auf dieser Seite zu beginnen.

\section{Mobilisation}

Stellt man beim Anlegen eines Tunnels fest, dass die Spannung auf der Schleimhaut zu hoch wird, sollte eine vertikale Chondrotomie zwischen Lamina perpendicularis und Lamina quadrangularis mit einem Knorpelritzmesser durchgeführt werden. Reicht diese Maßnahme nicht aus, um die Spannung auf die Schleimhaut zu vermindern, sollte zusätzlich eine horizontale Chondrotomie entlang der knorpeligen Basis des Septums im Übergang zur Prämaxilla erfolgen. Dabei werden die Schnittführungen der beiden Chondrotomien miteinander verbunden. Spätestens zu diesem Zeitpunkt muss dann auch der Fixpunkt der kaudalen Septumkante an der Spina nasalis anterior scharf getrennt werden. Damit ist eine vollständige Mobilisation des knorpeligen Septums erreicht. Da dieses nun nur noch mit den beiden Seitenknorpeln verbunden ist („septodorsaler Komplex“), kann es sich jetzt in 2 Richtungen bewegen. Einerseits ist aus der Mittellinie heraus eine Bewegung wie bei einer Tür („swinging door“), andererseits ist auch eine Einwärtsbewegung um den Rotationspunkt der Keystone-Area („K-Area“, ebenfalls 


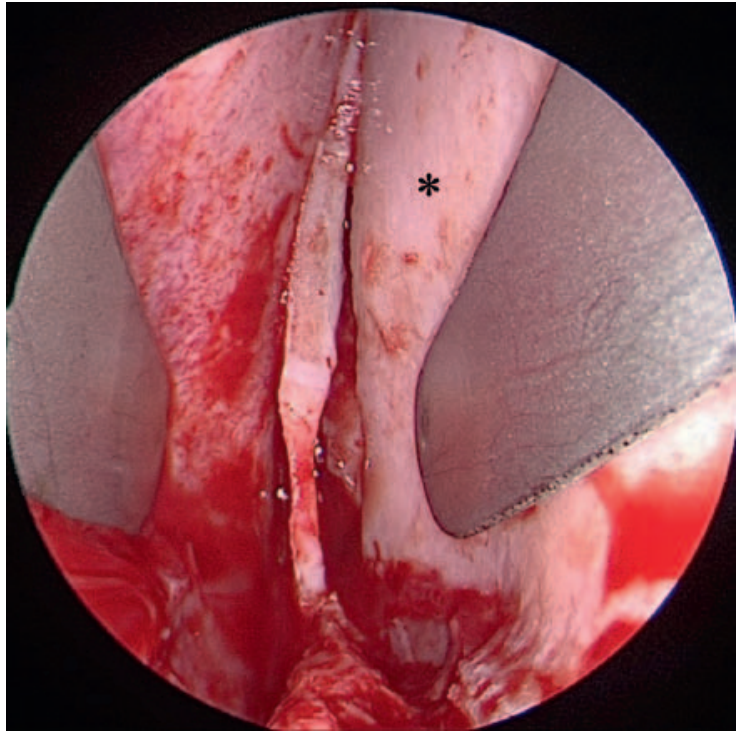

- Abb. 3 Intraoperativer Situs mit Anlage einer „swinging door" ${ }^{*}$ ) nach Durchführung der vertikalen und horizontalen Chondrotomie und Ablösen der kaudalen Septumkante von der Spina nasalis anterior.

ein Fixpunkt des Septums) am Übergang zwischen knorpeligem und knöchernem Nasenrücken möglich ( Abb. 3).

Auch der Knochen der posterioren Septumabschnitte, die Lamina perpendicularis und der Vomer, muss im Regelfall durch Osteotomien beweglich gemacht werden, um die Spannung auf die Schleimhaut zu minimieren oder um Deviationen zu entfernen. Geeignete Instrumente sind der 4mm-Septummeißel oder die „übersetzte“ Knochenschere.

\section{Resektion}

Die gut exponierten Septumareale lassen sich nun hinsichtlich relevanter Deviationen suffizient beurteilen. Unter „relevanten Deviationen“ werden Engstellen in der Nasenhaupthöhle verstanden, die den Luftweg deutlich einengen und nur noch einen schlitzförmigen Spalt zur Inspiration belassen. Da sich die engsten Abschnitte der Nase physiologisch zwischen Naseneingang und Nasenklappenregion befinden, muss diesem Areal erhöhte Aufmerksamkeit bei der Begradigung von Septumdeviationen geschenkt werden ( $\triangleright$ Abb. 4).

Zuerst empfiehlt sich die Kontrolle der korrekten Position der kaudalen Septumkante in Bezug auf die Spina. Häufig ist diese seitlich von der Spina abgerutscht und somit aus der Mittellinie verlagert, meistens verursacht durch ein Trauma oder eine zu hohe Knorpelspannung. In solchen Fällen muss der Fixpunkt scharf getrennt werden. Die isolierte Septumkante wird nach Reduktion der Spannung durch eine horizontale Chondrotomie - anschließend wieder mit der Spina verbunden. Hierzu wird eine resorbierbare 8er-Naht (Vicryl 3/0) angelegt. Die Verankerung

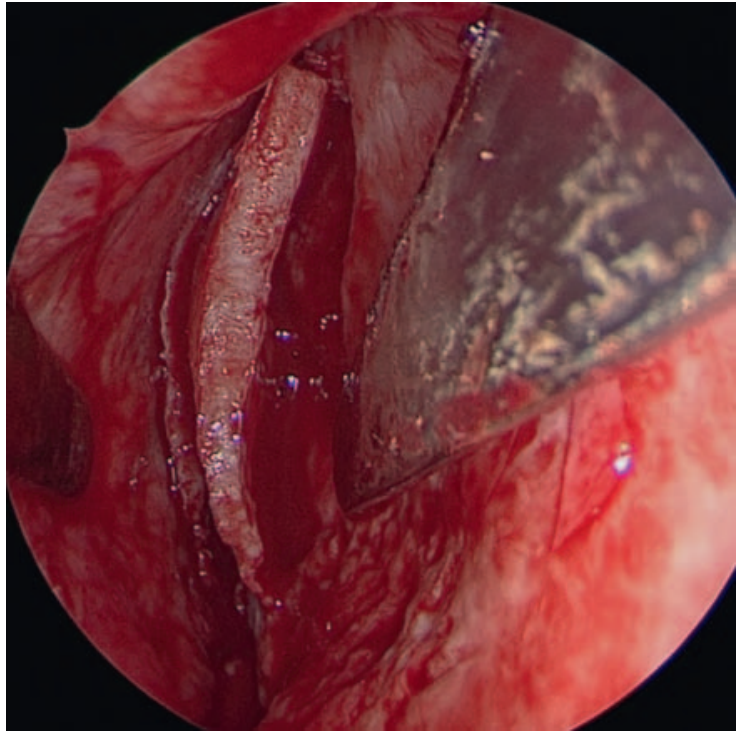

Abb.4 Intraoperativer Situs mit Blick auf eine hohe Deviation nach rechts.

funktioniert nur, wenn genügend periostale Fasern an der Spina erhalten werden ( $\triangleright$ Abb.5).

Danach sollte die Tragfähigkeit der kaudalen Kante überprüft werden. Erscheint diese zu strukturschwach, muss eine Verbesserung der Stabilität durchgeführt werden. Eine geeignete Lösung ist die Adaptation eines Strukturtransplantats aus autologem Septumknorpel, der „batten graft“. Dieser wird als Verbundtransplantat passgenau auf die Septumkante mit 3 resorbierbaren Matratzennähten (Vicryl 4/0) fixiert. Der batten graft sollte etwa so hoch sein wie die kaudale Kante und eine Breite von 5-8 mm aufweisen ( $\triangleright$ Abb. 6). Diese Maßnahme dient der langfristigen Stabilität und sollte im Sinne der Nachhaltigkeit einer SPL gesehen werden.

Merke

Besonders die hohe Deviation der Lamina perpendicularis führt zur Einengung und Verkleinerung des Nasenklappenwinkels. Wird sie nicht erkannt und entfernt, ist das häufig der Grund für eine Revisions-SPL.

Es wird deshalb empfohlen, nach Resektion von Knorpel und Knochen intraseptal die Nasenhaupthöhle besonders auf Höhe der Nasenklappe erneut hinsichtlich der Spaltraumerweiterung zu überprüfen. Mit der übersetzten Becker-Caplan-Schere lässt sich der Knochen Schritt für Schritt verkürzen, ohne dass die Stabilität der unmittelbar darüber befindlichen Keystone-Area gefährdet wird.

Die Nasenhaupthöhle erweitert sich nach posterior, sodass nur gravierende Luftpassage-Hindernisse begradigt werden sollten. Ein klassisches Beispiel dafür ist ein ausladender Vomersporn, der Kontakt zur unteren oder mittleren Nasenmuschel hat. 

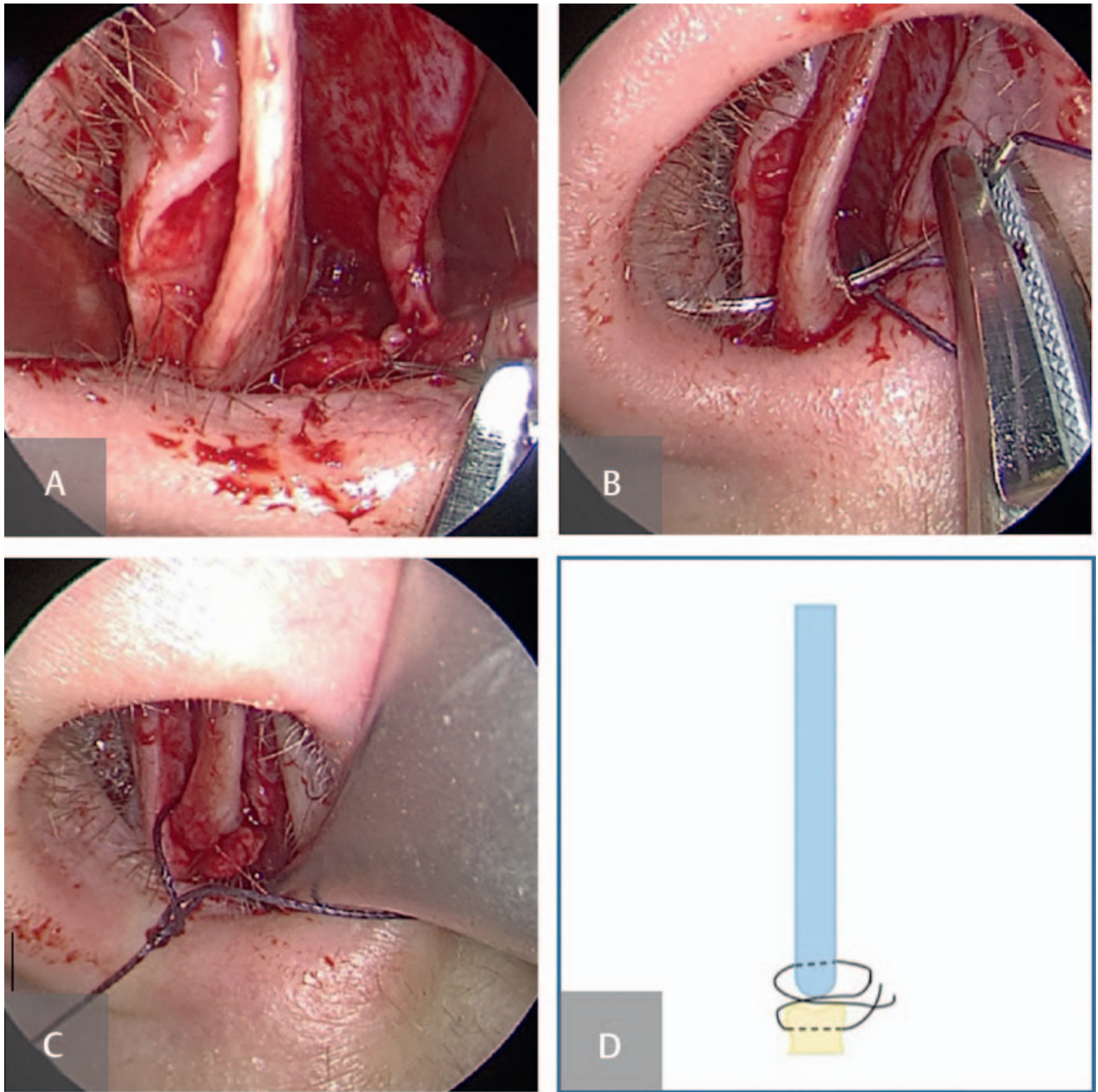

- Abb. 5 Durchführung der Spina-Naht. A Durchstich durch periostale Fasern der Spina nasalis anterior; B Durchstich durch die kaudale Septumkante; $\boldsymbol{C}$ Verknoten der 8er-Naht; D Schema der Spina-Naht.

\section{Merke}

Während der Resektion muss darauf geachtet werden, dass jederzeit ein stabiler Knorpelrahmen im Bereich der Lamina quadrangularis erhalten bleibt. Eine Schwächung des Rahmens führt in der Folge der Wundheilung und Narbenbildung zu den bekannten Stigmata der nicht korrekt durchgeführten SPL: knorpeliger Sattel („Pseudohöcker“), Retraktion der Kolumella, Absinken und Instabilität der Nasenspitze, Verbreiterung der Nasenspitze, quer-ovale bis runde Nasenlöcher.

Die letzten Schritte umfassen begradigende Maßnahmen an der Prämaxilla. Diese Zone lässt sich kombiniert über einen vorderen oberen und vorderen unteren Tunnel ex- ponieren. Der Einstieg in den vorderen unteren Tunnel gelingt nach Darstellung der nachfolgenden Landmarken: Spina nasalis anterior und Crista der Apertura piriformis. Eine scharfe Präparationstechnik mit der Schere oder dem Cottle-Messer ist bei der Durchtrennung der periostalen Fasern nützlich. Danach gelangt man auf den Nasenboden und befindet sich im unteren Tunnel. Es ist ratsam, nun die prämaxilläre Leiste tangential mit dem $4 \mathrm{~mm}$-Septummeißel abzutragen, um diese dann mit der Schere von der Mukosa zu trennen und zu resezieren. Eine mögliche arterielle Blutung aus der A. incisiva muss mit der HF-Kaustik oder mit Knochenwachs versorgt werden, um ein Septumhämatom zu vermeiden. 


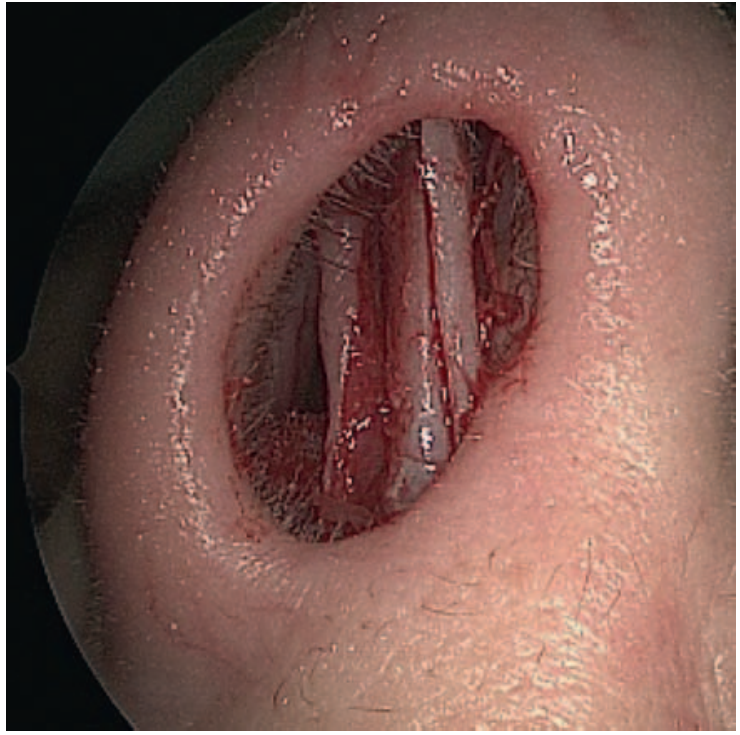

- Abb. 6 Intraoperativer Situs mit einem batten graft zur Stabilisierung der kaudalen Septumkante.

\section{Replantation und/oder Rekonstruktion}

Vorab wird in die beiden Nasenhaupthöhlen je eine Schwämmchen-Tamponade eingelegt. Dies dient der Stabilisierung der extrakorporal begradigten und replantierten Knorpel- und Knochenfragmente. Sie werden in anatomisch korrekter Position platziert. Posterior wird Knochen eingestellt, anterior Knorpel. Überlappungen der Fragmente sind zu vermeiden.

Es wird nochmals auf die zentrale Bedeutung einer strukturstabilen und adäquat hohen kaudalen Septumkante hingewiesen. Diese muss in einer „balancierten Spannung" ausgerichtet sein. Eine zusätzliche und vor allem nachhaltige Stabilität lässt sich durch Adaption des zuvor beschriebenen batten grafts erzielen. Eine ausreichende Höhe kann durch die Verwendung eines „caudal septal extension grafts" erreicht werden ( $\bullet$ Abb. 7).

Auch dieses Strukturtransplantat wird vorzugsweise aus autologem Septumknorpel gewonnen. Das Transplantat wird ebenfalls durch Nähte an der kaudalen Kante fixiert. Durch eine schrittweise Kürzung kann der Operateur unter optischer Kontrolle von der Seite die ästhetisch passende Höhe der Nasenspitze festlegen. Eine Überlänge in ventraler Richtung, wie in $>$ Abb. 7 erkennbar, ermöglicht zudem den Ausgleich einer retrahierten Kolumella.

Vor dem Verschluss der Inzision sollte abschließend nochmal die korrekte Verbindung zwischen Spina nasalis anterior und kaudaler Septumkante mit der AdsonBrown-Pinzette überprüft werden. Dazu fasst die Pinzette den Septumknorpel und versucht durch dosierten Druck eine Einwärtsbewegung zu erzielen. Gelingt dies nicht, ist die angelegte 8er-Naht effizient.

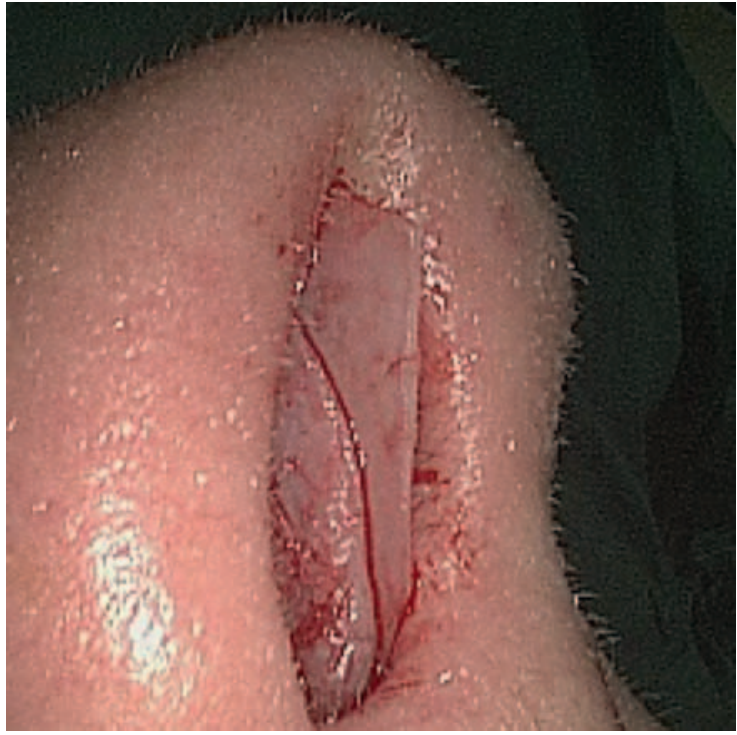

- Abb. 7 Intraoperativer Situs mit einem „caudal septal extension graft“.

\section{Naht der Inzision und Fixierung}

Abschließend wird die Hemitransfixionsinzision mit einer resorbierbaren Naht (z. B. Vicryl 4/0 P3) in Einzelknopftechnik verschlossen. Danach sollten Septumfolien zur temporären Stabilisierung und Hämatom-Prophylaxe mit einem monofilen, nicht resorbierbaren Faden (z. B. Prolene 3/0) eingenäht werden. Diese können etwa nach 4-7 Tagen ambulant entfernt werden. Eine vordere Tamponade der Nase für eine Nacht wird empfohlen, ist aber nicht obligatorisch. Werden Tamponaden verwendet, so müssen diese am Nasenrücken mit einem Pflaster sicher fixiert werden, um eine akzidentelle Dislokation mit Gefahr der Aspiration zu verhindern.

\section{Komplikationen}

Die beschriebene Technik der SPL ermöglicht im Regelfall einen komplikationsarmen postoperativen Verlauf. Sollten dennoch Komplikationen auftreten, so können diese in Restdeformitäten, Infektionen und dem Auftreten einer Septumperforation subsummiert werden.

Restdeformitäten des Septumknorpels resultieren in einer persistierenden Nasenatmungsbehinderung, können aber auch sichtbare ästhetische Veränderungen der Nasenbasis und des Nasenrückens verursachen. Eine intraseptale Infektion, häufig aus einem Hämatom resultierend, ist der Septumabszess. Beide Komplikationen müssen möglichst zügig operativ entlastet werden, um eine Nekrose des Knorpels zu vermeiden und - im Falle eines Abszesses ein Fortschreiten der Infektion nach intrakraniell zu verhindern. In diesem Fall muss bei der präoperativen Aufklärung auch an die Ohrknorpelentnahme gedacht werden. Sollte 
der Septumknorpel bereits zerstört sein, wird der Ohrknorpel als Ersatz transplantiert.

Die Septumperforation ist eine seltene postoperative Komplikation, wenn die Cottle-Technik der plastischen Septumkorrektur angewendet wurde. Behandelt werden nur symptomatische Perforationen. Therapie der Wahl ist der Septumperforationsverschluss unter Verwendung der 4-Brücken-Lappen Technik mit intraseptaler autologer Ohrknorpel-Interposition.

\section{KERNAUSSAGEN}

- Die Dimension, die Position und die Stabilität des knorpeligen Septums sind für den Erfolg einer SPL maßgeblich.

- Ziel der SPL ist die Schaffung ausreichend weiter Spalträume in den vorderen Nasenabschnitten, speziell im Bereich der Nasenklappenregion. Das knorpelige Septum muss dafür in einen Zustand der „balancierten Spannung“ überführt werden.

- Die wesentlichen Schritte der Septumplastik umfassen die Inzision (= Hemitransfixion), die Bildung der subperichondralen und/oder subperiostalen Tunnel, die Mobilisation durch Chondrotomien und/oder Osteotomien, die Resektion von Knorpel und/oder Knochen, die Replantation von extrakorporal begradigtem Material/Rekonstruktion des kaudalen Septums und schließlich die Naht der Inzision und Fixation mittels Septumfolien.

- Die Festigkeit der kaudalen Septumkante ist für die Nachhaltigkeit der Operation entscheidend und gewährleistet die Stabilität der gesamten knorpeligen Nase für Jahrzehnte.

\section{Wissenschaftlich verantwortlich gemäß Zertifizierungsbestimmungen}

Wissenschaftlich verantwortlich gemäß Zertifizierungsbestimmungen für diesen Beitrag ist Prof. Dr. med. Marc Scheithauer.

\section{Interessenkonflikt}

Erklärung zu finanziellen Interessen

Forschungsförderung erhalten: nein; Honorar/geldwerten Vorteil für Referententätigkeit erhalten: nein; Bezahlter Berater/interner Schulungsreferent/Gehaltsempfänger: nein; Patent/Geschäftsanteile/Aktien (Autor/Partner, Ehepartner, Kinder) an Firma (Sponsor der Veranstaltung): nein; Patent/ Geschäftsanteile/Aktien (Autor/Partner, Ehepartner, Kinder) an Firma (Nicht-Sponsor der Veranstaltung): nein.

Erklärung zu nichtfinanziellen Interessen

Die Autorinnen/Autoren geben an, dass kein Interessenkonflikt besteht.

\section{Autorinnen/Autoren}

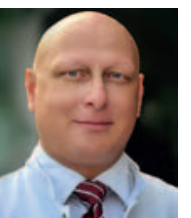

\section{Marc Scheithauer}

Leitender Oberarzt der Klinik für Hals-NasenOhrenheilkunde, Kopf- und Halschirurgie, Universitätsklinik Ulm.

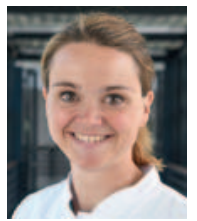

\section{Janina Hahn}

Assistenzärztin der Klinik für Hals-NasenOhrenheilkunde, Kopf- und Halschirurgie, Universitätsklinik Ulm.

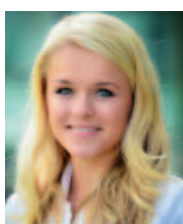

\section{Marlene Corinna Cosima Wigand}

Fachärztin der Klinik für Hals-Nasen-Ohrenheilkunde, Kopf- und Halschirurgie, Universitätsklinik Ulm.

Korrespondenzadresse

Prof. Dr. med. Marc Scheithauer

Klinik für Hals-Nasen-Ohrenheilkunde, Kopf-Halschirurgie, Universitätsklinikum Ulm

marc.scheithauer@uniklinik-ulm.de

\section{Literatur}

[1] Theissing J, Rettinger G, Werner JA. ENT-Head and Neck Surgery: Essential Procedures. 4th ed New York: Thieme; 2006

[2] Huizing EH, de Groot JAM. Functional Reconstructive Nasal Surgery. 2nd ed New York: Thieme; 2015

[3] Behrbohm H, Tardy ME. Funktionell-ästhetische Chirurgie der Nase. 1. Aufl Stuttgart: Thieme; 2004

[4] Mlynski G, Pirsig W. Funktionell-ästhetische Rhinochirurgie unter besonderer Berücksichtigung physiologischer Aspekte. 1. Aufl Stuttgart: Thieme; 2018

[5] Cottle MH, Loring RM. Surgery of the nasal septum; new operative procedures and indications. Ann Otol Rhinol Laryngol 1948; 57 (3): 705-713. doi:10.1177/000348944805700309

[6] Cottle MH, Loring RM. Newer concepts of septum surgery, present status. Eye Ear Nose Throat Mon 1948; 27 (9): 403-406

[7] Rettinger G, Kirsche HP. Complications in Septoplasty. Facial Plast Surg 2006; 22 (4): 289-297

\section{Bibliografie}

Laryngo-Rhino-Otol 2020; 99: 733-740

DOI 10.1055/a-1021-2297

ISSN 0935-8943

(C) 2020. Thieme. All rights reserved.

Georg Thieme Verlag KG, Rüdigerstraße 14,

70469 Stuttgart, Germany 


\section{Punkte sammeln auf CME.thieme.de}

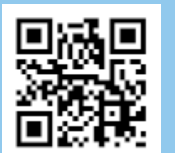

Diese Fortbildungseinheit ist in der Regel 12 Monate online für die Teilnahme verfügbar.

Den genauen Einsendeschluss finden Sie unter https://eref.thieme.de/CXDWV7S.

Sollten Sie Fragen zur Online-Teilnahme haben, finden Sie unter https://cme.thieme.de/hilfe

eine ausführliche Anleitung. Wir wünschen viel Erfolg beim Beantworten

der Fragen!

Unter https://eref.thieme.de/CXDWV7S oder über den QR-Code kommen Sie direkt zur Startseite des Wissenstests.

VNR 2760512020158724514

\section{Frage 1}

Welche 3 Landmarken sollten nach der korrekten Durchführung einer Hemitransfixionsinzision erkennbar sein?

A kaudale Septumkante, Proc. sphenoidalis, Prämaxilla

B Lamina perpendicularis, Spina nasalis anterior, Crista der Apertura piriformis

C Vomer, kaudale Septumkante, Prämaxilla

D kaudale Septumkante, Spina nasalis anterior, anterior-inferiorer Septumwinkel

E Crista der Apertura piriformis, anterior-inferiorer Septumwinkel, Proc. sphenoidalis

\section{Frage 2}

Welche Aussage zum „batten graft" trifft nicht zu?

A Er kann zur Verbesserung der Stabilität der kaudalen Septumkante eingenäht werden.

B Er wird mit ca. 3 resorbierbaren Matratzennähten fixiert.

C Es handelt sich um ein Strukturtransplantat aus allogenem Septumknorpel.

D Die Maßnahme wird im Rahmen der Nachhaltigkeit einer SPL gesehen.

E Der batten graft sollte eine Breite von 5-8 mm aufweisen.

\section{Frage 3}

Welche Aussage zu vermeidbaren Komplikationen der Septumchirurgie trifft nicht zu?

A Eine Blutung aus der A. incisiva sollte zur Prophylaxe eines Septumhämatoms mit Knochenwachs und/oder HF-Kaustik versorgt werden.

B Eine hohe Deviation der Lamina perpendicularis im Bereich des Nasenklappenwinkels sollte belassen werden, um die Stabilität des Knorpelrahmens nicht zu gefährden.

C Stigma einer nicht korrekt durchgeführten SPL ist u. a. eine Retraktion der Kolumella.

D Eine weitere Maßnahme zur Septumhämatom-Prophylaxe ist die Einnaht von Septumfolien.

E Die temporäre Einlage einer vorderen Nasentamponade wird empfohlen, sie sollte als Aspirationsschutz am Nasenrücken fixiert werden.

\section{Frage 4}

Welche anatomische Struktur hat nach Cottle die wesentliche Stützfunktion des Septums?

A die knorpelige Lamina quadrangularis

B die knöcherne Lamina perpendicularis

C der Vomer

D der Nasenklappenwinkel

E die knöcherne Lamina quadrangularis

\section{Frage 5}

Welche Aussage zur Durchführung der Mobilisation der septalen Strukturen trifft zu?

A Die horizontale Chondrotomie zwischen Lamina perpendicularis und Lamina quadrangularis sollte mit dem Knorpelritzmesser durchgeführt werden.

B Der Fixpunkt der kaudalen Septumkante an der Spina nasalis anterior darf zum Erhalt der Spannung nicht durchtrennt werden.

C Für die Durchführung einer vertikalen Chondrotomie wird die Anwendung einer übersetzten Schere empfohlen.

D Eine „swinging door“ ist ein Zeichen übermäßiger Mobilität und sollte dementsprechend vermieden werden.

E Der weiter posterior gelegene Vomer und die Lamina perpendicularis müssen normalerweise durch Osteotomien mobil gemacht werden.

\section{- Weitere Fragen auf der folgenden Seite...}




\section{Punkte sammeln auf CME.thieme.de}

\section{Frage 6}

Welche Aussage zur Nachhaltigkeit der SPL trifft nicht zu?

A Eine Verbesserung der Projektion der Nasenspitze kann durch Verwendung eines „caudal septal extension grafts“ erreicht werden.

B Vor Verschluss der Hemitransfixionsinzision sollte die korrekte Verbindung zwischen Spina nasalis anterior und kaudaler Septumkante mit der Adson-Brown-Pinzette überprüft werden.

C Eine resorbierbare 8er-Naht (Vicryl 3/0) wird zur Refixierung der kaudalen Septumkante empfohlen.

D Die extrakorporal begradigten Knorpel- und Knochenfragmente sollten zum Erreichen einer höheren septalen Stabilität überlappend replantiert werden.

E Bei Durchführen der Hemitransfixionsinzision darf der Knorpel nicht inzidiert werden, um die Integrität der kaudalen Septumkante nicht zu gefährden.

\section{Frage 7}

Die wesentlichen Schritte der Septumplastik lauten in korrekter Reihenfolge:

A Inzision - Mobilisation - Resektion - Tunnelung - Replantation - Verschluss der Hemitransfixion

B Zugang - Resektion - Hemitransfixion - Mobilisation - Replantation - Naht

C Hemitransfixion - Zugang - Mobilisation - Resektion - Replantation - Naht und Fixation

D Mobilisation - Zugang - Hemitransfixion - Resektion - Fixation - Replantation

E Inzision - Tunnel - Resektion - Mobilisation - Fixation Replantation

\section{Frage 8}

Zu den Stigmata der nicht korrekt durchgeführten SPL gehört/ gehören nicht:

A quer-ovale bis runde Nasenlöcher

$B$ ein Pseudohöcker

C die Verschmälerung der Nasenspitze

D die Retraktion der Kolumella

E das Absinken der Nasenspitze

\section{Frage 9}

Welche Funktion erfüllt ein „caudal septal extension graft“?

A Verbreiterung eines zu schmalen Nasenrückens

B Stabilisierung des kaudalen Septums und Verbesserung der Projektion

C Verbreiterung von zu schmalen Nasenlöchern

D Überhöhung einer knorpeligen Sattelnase

E Öffnen der Nasenklappe

\section{Frage 10}

Welche anatomischen Strukturen definieren den „septodorsalen Komplex"?

A Septum und Processus frontalis maxillae

B Flügelknorpel, Seitenknorpel und Septum

C Seitenknorpel und Flügelknorpel

D Septum, Seitenknorpel und Processus frontalis maxillae

E Septum und Seitenknorpel 